\title{
Forum
}

\section{'Analysis of Radar PAD Information and a Suggestion to Reshape the PAD'}

\author{
from J. P. O'Sullivan \\ (Sperry Marine Ltd)
}

I. INTRODUCTION. Wu Zhao-lin's paper (p. 124) pays tribute to the fact that the situation evaluation strengths of the Sperry Marine 'Predicted Area of Danger' (PAD) display convention promotes a more rapid and comprehensive understanding of collision risk than might be achieved using time-variable vector formats.

The author is of the opinion that specially directed training programs will be required to optimize the benefits of the PAD convention. He concludes, furthermore, that the effectiveness of the convention could be improved by changing the shape of the PAD symbol. He comments on an interesting aspect of possible ARPA influence on future amendments to the Collision Regulations.

The paper represents an independent assessment of an aspect of ARPA technology and deserves attention.

2. ANALYSIS OF PAD DISPLAY. An excellent description of the PAD symbol and its information content is offered at the start of the $\mathrm{Wu}$ Zhao-lin paper.

Two comments may augment the author's assessment. First, the CPA-linked PAD symbol contains a permanent allowance for sensor and system errors. Hence, adopting a heading for ownship that is tangential to a PAD symbol when in steady motion will result in a CPA distance somewhat in excess of the pre-selected CPA control established. Secondly, the PAD symbol provides a graphic prediction on the PPI of the area in which a 'close quarter situation' for a particular target will occur. This accomplishment remains unique in ARPA technology.

The author states that manoeuvring ownship with respect to PAD symbols acting as obstructions along its intended trackline does not provide a clear indication of whether ownship will pass ahead or astern of the target. At first glance, this statement is correct, but requires further examination. There are complexities in encounters with faster ships which are emphasized correctly by the PAD technique.

The author provides a detailed, systematic and worthy mathematical analysis of many possible PAD configurations, an approach likely to have been influenced by the work of the late Captain K. D. Jones of Liverpool Polytechnic, Department of Maritime Studies.

Mathematical analysis, however, requires to be tempered with practical operating advice addressed to the navigator.

Wu Zhao-lin emphasizes the important relationship between PAD geometry and actual and predictive relative motion data flowing from the radar sensor. This bias towards relative motion as the basis for prediction of motion and for manoeuvre planning is most significant, and is in keeping with IMO recommendations. The approach protects the ARPA from the extremes of error influences and provides the navigator with means for independent verification of the ARPA information.

The author's Fig. I represents a specific encounter with a slower target. It will be 
seen that the actual relative track for the encounter lies outside the sector of predicted relative tracks for inadequate CPA. Hence, the PAD symbol lies off the heading marker.

The dynamics and the geometry of the actual encounter establish the along-track terminations of the PAD symbol, as illustrated. The across-track components of the symbol, on the other hand, are related to the pre-selected CPA distance value alone and are the same for all targets in track.

The different regimes that determined the two axes of the PaD symbol led Sperry Marine, after much investigation and trials in the early 1970s, to select an ellipse as an appropriate symbol with which to depict risk. The hexagonal symbol used today is merely a digital representation of the ellipse.

3. PAD CATEGORIES. The author's classification of PADS lists 10 distinct categories of symbol configurations. Examination of the analysis process he has used reveals some duplication, which will permit a degree of simplification.

Sperry Marine would suggest that, in practical terms, there are only three distinct PAD categories that the navigator must consider:

(i) Targets producing a single PAD. The following categories of targets will produce a single PAD symbol: (a) a slower or equi-speeded target whose PAD is within display range; (b) a faster target showing a merged PAD as it approaches its CPA position [with a PPC 'Point of Possible Collision' or, in the later stages as CPA is approached, without a PPC]; (c) a faster target crossing or approaching head-on during the early stages of the encounter, when the second PAD is likely to be beyond display range.

(ii) Duplex PADs for faster targets. Overtaking targets, in particular, produce distinctive duplex PADs from the moment of acquisition.

(iii) Targets without PADS. Targets which display a true vector only without a PAD, thus categorized as 'non-hazardous,' are classified as follows: (a) a faster target which has passed through its CPA position; or (b) a slower target whose PAD is outside the display range.

Thus a target is capable of producing one or two PADs or may fail to produce any. This variation has been the cause for concern on many occasions.

These phenomena exist in the real world and are not a by-product of the PAD convention. Collision-avoidance literature cites the occasional failure of the stratagem of making broad alterations of heading when presented with steady compass-bearing situations.

The subdivisions within category (i), particularly, and within category (iii), to a lesser extent, require to be appreciated.

The principal parameters determining PAD classification are, first, the relative position of the target; secondly, the speed ratio of ownship and target vessel; and thirdly, a parameter for faster targets which can be described as a 'time into encounter' factor.

$\mathrm{Wu}$ Zhao-lin has stressed, correctly, that the results of passing close to either the primary or secondary PADs of a faster target showing both PADs or the results of passing close to a single PAD, depending on whether it has been established by a faster or slower target, is subject to a manoeuvring ambiguity.

If the target is slower than ownship, the PAD is a primary PAD and it has a distinct and invariable manoeuvre consequence: passing behind the PAD, i.e. passing between the PAD and the target creating it, results in passing behind the target and vice versa. This can be considered to be the nominal encounter case. Where time and manoeuvre room exist and legislation permits it, this is the optimum manoeuvre to conduct.

There is no change in this rule as it applies to the consequences of manoeuvring in respect to the primary PAD of a faster target.

It follows from the primary PAD manoeuvring rule that if ownship elects to pass between the duplex PADs of certain faster targets, the resultant relative track will be 
directed astern and ownship will cross ahead of the target. This is a manoeuvre that should be undertaken only after careful consideration of all the circumstances of the encounter and the traffic situation.

Conversely, whenever ownship decides to remain clear outside both PADs of a faster target, the relative track will pass ahead of ownship. Hence, if the dual PADs of a faster target have merged, ownship has only one option available : to assist the target clear away ahead by avoiding the merged PAD.

An alternative visualization of this primary PAD rule, considering the slower-crossing target of the author's Fig. I, can be derived by means of differentiating from the worst-case situation, which is to adopt a heading towards the centre of the PAD and hence towards the PPC. Ownship's present heading (in front of the PAD) in Fig. I represents an alteration away from the predicted collision point that must result in the target passing astern.

It follows, therefore, that passing between any PAD and the target creating it can have only one result : the target will pass ahead of ownship.

The ambiguity that exists, therefore, lies in determining the result of passing in front of a single PAD, the classification of which has not been determined, i.e. whether the PAD is a primary PAD (faster or slower target) or a merged PAD of a faster target.

In practice, there should be no difficulty in resolving this ambiguity. A hypothetical three-target encounter is illustrated in Figure A. Targets $I$ and 3 are faster than ownship and their PADs are drawn in firm lines; target 2 is slower and its PAD is drawn as a hatched symbol, to distinguish it from the others.

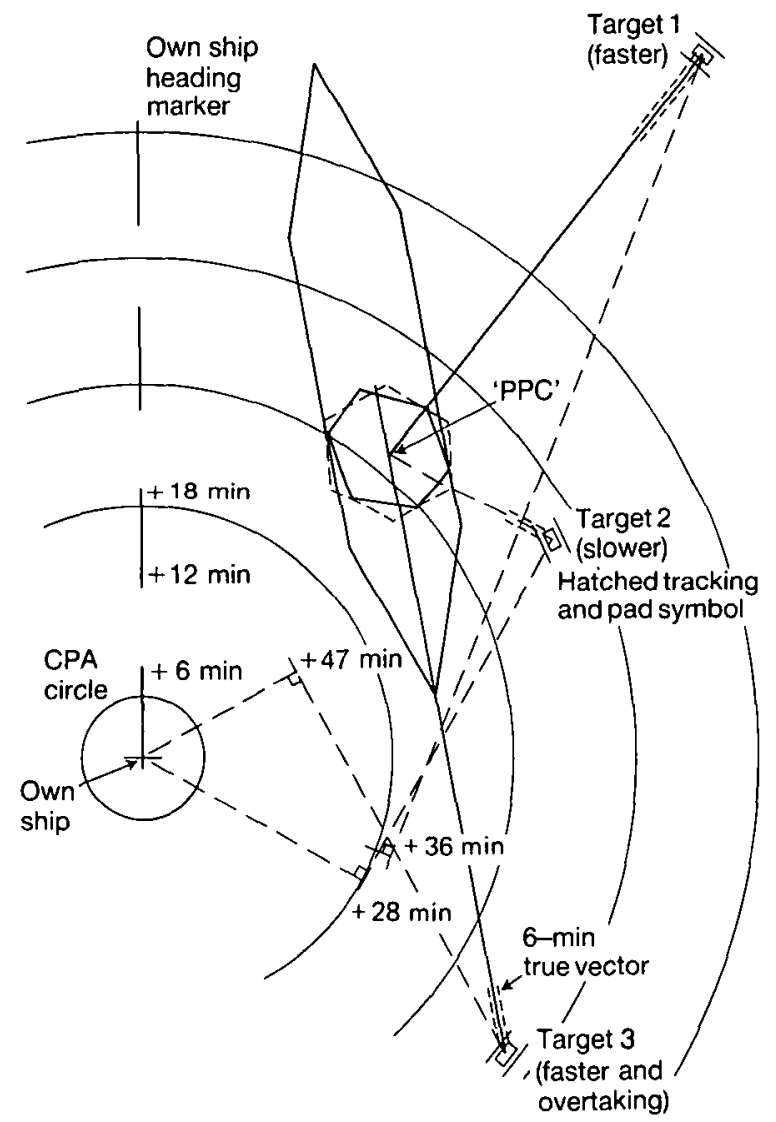

Fig. A. Classification of a single PAD 
The PADs for targets 1 and 2 are co-located, have similar proportions and result in the same sense and general value of CPA. They both obey the nominal manoeuvre rule. Target 3 , on the other hand, represents an overtaking target, the dual PADs of which have merged already. (This target is at a stage in its encounter when the dual pPCs are also merging.)

It can be seen that the special case of the merged dual PAD is clearly identifiable in terms of its distinctive elongated shape, enabling the ambiguity of the result of passing in front of its PAD to be anticipated.

4. REDUCING CPA CONTROL IN OPERATIONS. The author discusses reducing the pre-selected $C P A$ distance as a means of reducing the area of coverage of the PAD symbol, as though this would create an impression that the danger level had been reduced. This observation leads him to conclude that different categories of encounters should have different cPas assigned to them.

Sperry Marine has advised consistently that once a suitable CPA selection has been made, it should not be reduced in a casual manner thereafter. This procedure could well be irresponsible and tantamount to establishing false standards of safety.

Providing variable CPA values in respect to different categories of target risk is not an approach that Sperry Marine would support at this stage of the technology. It could lead to some complexity and possible confusion, and would make interpretation less than straightforward.

5. SUGGESTED INFLUENCE OF PADS ON COLREgS. The author discusses potential situations in which PADs identify safe manoeuvres adequately which are expressly prohibited by the last amendment to the COLREGS and which could pose a problem for the navigator.

These are original views and are in keeping with opinions held by Sperry Marine. It was perhaps unfortunate that those changes in the COLREGS were legislated at a time when operating experience with ARPA had not been gained.

These views, therefore, represent valuable critical statements, the publication of which may encourage a debate which will be beneficial to the industry.

6. THE SUGGESTED CHANGES TO PADS. The author's recommended change (to show a ship-shaped PAD symbol) is not acceptable, however. It would be an improvement in the case of the primary PAD only.

7. ANTICIPATING THERESULTS OFTARGETMANOEUVRES. A sccondary change that the author proposes (displaying a segment of the locus of the PPC outside the PAD) appears to be based on the opinion that it would serve as an aid to forecasting the results of possible target manoeuvres.

While an appreciation of the likely results of target manoeuvres is beneficial and it is known that experienced users become capable of making those judgements, the display of the PPC locus on either side of the PAD would be a complication which, on balance, would not gain much advantage.

Collision-avoidance manoeuvres undertaken in respect of the sailing rules must, of necessity, be based on the premise that the target will maintain its steady motion. Attempts of a decade ago to develop an aeronautical C/A system which would encompass all possible manoeuvre actions of target aircraft were abandoned, though there is some indication that investigation has been reactivated.

The author is to be complimented on his perceptive understanding of the fundamental dynamics of the PPC: the coaxial circle theory of Apollonius as applied by R. F. Riggs of Sperry Marine to collision avoidance.

Sperry Marine welcomes the publication of this paper in the Journal as an encouragement of a worthy research effort. 